\title{
Research on High-Frequency Vibration Isolation for Rod-Shaped Structures Based on One-Dimensional Phononic Crystals
}

\author{
Yi Zhou *, Dongxu Li \\ National University of Defense Technology, Changsha, Hunan, China \\ ”gfkd_zhouyi@126.com
}

Keywords: rod-shaped structures; high-frequency; vibration isolation; one-dimensional phononic crystals

\begin{abstract}
The phononic crystal (PC) has band gap characteristics, which can be used to design the band gap range by its microstructure, realizing the vibration suppression of specific frequency bands. Therefore, based on one-dimensional (1D) PCs, this paper proposes an approach to high-frequency vibration isolation for rod-shaped structures. First, the model of bipropellant rod-shaped structures of 1D PCs is established, and the transfer matrix method (TMM) of band gap calculation is introduced. Then, the characteristics of band gap and their influence factors are studied using TMM. And combined with the frequency response curves, effect of boundary conditions on finite bipropellant rod of 1D PCs is also studied. Last, taking the truss rod of support structure of space camera as an example, simulation is conducted. Results show that the proposed method is effective with great vibration suppression of $750-2000 \mathrm{~Hz}$, broadening the application prospects in other areas.
\end{abstract}

\section{Introduction}

Many components on spacecrafts are rods, such as the support truss of space camera[1], the tripod of solar array structures[2], the truss structure of the Space Station[3] and etc., as shown in Fig. 1. In the process of launch, separation, orbit operation and return, the vibration caused by the rocket thrust pulse, the separation shock, the micro vibration, the flutter and other inspiriting loads will propagate on these components, distroying the sturctures or affecting the performance of other equipments on spacecrafts. Up to now, passive vibration isolation method and active vibration control method are the main approaches to vibration reduction for spacecrafts. But the passive vibration isolation method relys on the stiffness and damping of the isolator, limited by the characteristics of the damping material itself. And the devices of active vibration control are always hurg and complexed, which might not be reliable in severe space environment. On the contrary, the band gap characteristics of PCs can be adjusted simply by the design of the microstructure configuration to cover the frequency range of interest. Moreover, the wide band gaps do not depend on the properties of materials and the structure of vibration suppression is more reliable.

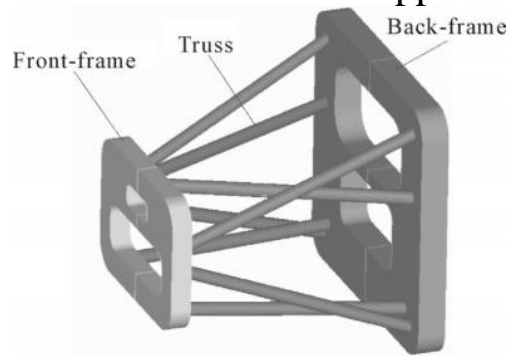

(a) Support truss of space camera

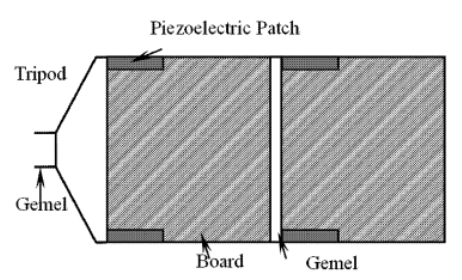

(b) Tripod of solar array sturctures

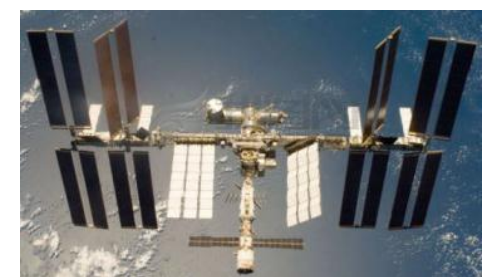

(c) Truss structure of the Space Station

Figure.1 Rods on spacecraft

PC [4] is actually a kind of periodic material, whose remarkable characteristic is band gap. Recent research[5][6] show that for local resonant PCs[7], when the vibration frequency gets close to the natural frequency of microstructure, the elastic wave resonates locally with the microstructure(the 
minimun unit cell of PCs), consuming the energy of vibration and suppressing the propagation of vibrtion waves, which can get application in acoustic wave control[8], noise and vibration reduction[9] and other areas, but has not been used in high-frequency vibration reduction for rod-shaped structures on spacecrafts. Theoratically, by using components of PCs as structural rods instead of traditional ones, the capacity on vibraition suppression of components can be increased. 1D PCs have a simple structure configuration, easy fabrication and wide band gap, making it most possible to get application in engineering first.

Given all this, this paper proposes an approach to high-frequency vibration isolation for rod-shaped structure based on 1D PCs. The model of bipropellant rod-shaped structure of 1D PCs is established, and the band gap characteristics of both infinite bipropellant rod-shaped structures and finite rods of 1D PCs are studied using TMM. And taking the truss rod of support structure of space camera as an example, simulation is conducted validating the proposed method of high-frequecy vibration reduction.

\section{Characteristics of 1D PCs}

According to the theory on band gaps of the local resonant PCs, the band gap characteristics are closely related to the structure of the microstructure. Therefore, the 1D PC is studied this paper using TMM[10], and the variation of the band gap characteristics is analyzed. Then combined with the frequency response curves of the finite 1D PC rod, the research on its capacity of vibration suppress is carried out, which provides theoretical guidance for the design of high-frequency vibration isolation for rod-shaped structures based on 1D PCs.

Calculation theory of 1D PC gaps based on TMM[11]. 1D PCs[11][12] are usually considered as periodic structures consisting of rod-microstructures. And according to the number of kinds of materials in a microstructure, it can be divided into bipropellant ones, tripropellant ones, etc.. In this paper, the microstructure is made of two different materials, denoted as A and B with constant radius, as shown in Fig. 2(a). The lengths of rod A and B are respectively $a_{1}$ and $a_{2}$, thus the lattice constant $a=a_{1}+a_{2}$. The radius of both $\operatorname{rod} \mathrm{A}$ and $\mathrm{B}$ is $r$, and moreover, $a \geq 10 r . S$ represents the cross-sectional area. The density and elastic modulus of material $\mathrm{A}$ is $\rho_{1}$ and $E_{1}$, and $\rho_{2}$ and $E_{2}$ for material B. The $x$ axis is along the period direction of the rod-shaped structures. The $y$ axis is perpendicular to direction $x$ within paper. The $z$ axis is vertical to paper, thus composing the right hand coordinate system with $x$ and $y$ axes, as shown in Fig. 2(b). Theoretically, the perfect rod-shaped structures of 1D PCs is infinite along $x$ axis, but finite along the other two axes.

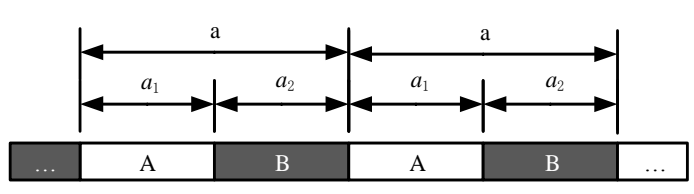

(a) phsical model

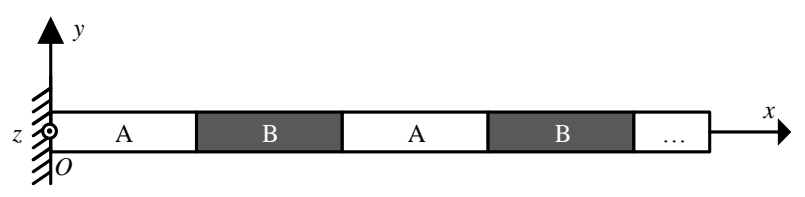

(b) coordinate system

Figure 2. Model of perfect bipropellant rod-sturctures of 1D PCs

The wave equation[13] of longitudinal vibration on a bar is

$$
\frac{\partial^{2} u}{\partial t^{2}}=c^{2} \frac{\partial^{2} u}{\partial x^{2}}
$$

where $u(x, t)$ is the longitudinal displacement at the position $x$ and the moment $t$, and $c=\sqrt{E / \rho}$ is the longitudinal wave velocity, $E$ the elasticity modulus, $\rho$ the density.

Assuming that

$$
u(x, t)=v(x) e^{-\mathrm{j} \omega t}
$$


where $v(x)$ is the longitudinal vibration amplitude at the position $x$, and $\omega$ is the angular frequency of longitudinal waves.

Substituting Eq. 2 into Eq. 1, then the amplitude $v(x)$ can be calculated as

$$
v(x)=A \cos (\lambda x)+B \sin (\lambda x)
$$

where $A, B$ are the unknown state parameters, and $\lambda=\omega / c$.

Thus, the stress at the position $x$ can be written as follows

$$
F(x)=E S v^{\prime}(x)=-E S \lambda A \sin (\lambda x)+E S \lambda B \cos (\lambda x)
$$

Considering a 1D PC rod microstructure composed of $m$ kinds of various materials, each microstructure can be divided into $m$ parts, and that each bar is made of uniform material. For every bar, $l_{i}$ is the length, $S_{i}$ the cross-sectional area, $E_{i}$ the elastic modulus, and $\rho_{i}$ the density. For microstructure $n$ bar $i$, the local coordinate system is established as shown in Fig. 3.

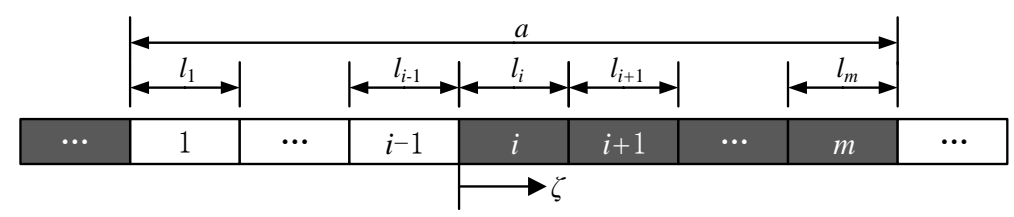

Figure 3. Local coordinate system of 1D PC rod

Note that $\mathbf{Q}_{i}^{n}(\zeta)=\left[\begin{array}{ll}v_{i}^{n}(\zeta) & F_{i}^{n}(\zeta)\end{array}\right]^{T}$ and $\mathbf{X}_{i}^{n}=\left[\begin{array}{ll}A_{i}^{n} & B_{i}^{n}\end{array}\right]^{T}$, thus

$$
\mathbf{Q}_{i}^{n}(\zeta)=\left[\begin{array}{cc}
\cos \left(\lambda_{i} \zeta\right) & \sin \left(\lambda_{i} \zeta\right) \\
-E_{i} S_{i} \lambda_{i} \sin \left(\lambda_{i} \zeta\right) & E_{i} S_{i} \lambda_{i} \cos \left(\lambda_{i} \zeta\right)
\end{array}\right] \mathbf{X}_{i}^{n}, \zeta \in\left[0, l_{i}\right] i=1,2, \cdots, m
$$

Considering the continuity of the right end of microstructure $n$ bar $i$ and the left end of microstructure $n$ bar $i+1$, there are

$$
\mathbf{Q}_{i}^{n}\left(l_{i}\right)=\mathbf{Q}_{i+1}^{n}(0), i=1,2, \cdots, m-1
$$

Thus

$$
\mathbf{X}_{i+1}^{n}=\mathbf{L}_{i+1}{ }^{-1} \mathbf{R}_{i} \mathbf{X}_{i}^{n}, i=1,2, \cdots m-1
$$

Similarly

$$
\mathbf{X}_{m}^{n}=\mathbf{L}_{m}{ }^{-1} \mathbf{R}_{m-1} \mathbf{L}_{m-1}{ }^{-1} \mathbf{R}_{m-2} \cdots \mathbf{L}_{2}{ }^{-1} \mathbf{R}_{1} \mathbf{X}_{1}^{n}
$$

Considering the continuity of the right end of microstructure $n$ bar $m$ and the left end of microstructure $n+1$ bar 1 , there are

$$
\mathbf{Q}_{m}^{n}\left(l_{i}\right)=\mathbf{Q}_{1}^{n+1}(0)
$$

Thus

$$
\mathbf{X}_{1}^{n+1}=\mathbf{L}_{1}^{-1} \mathbf{R}_{m} \mathbf{X}_{m}^{n}
$$

According to Eq. 10 and Eq. 8, then

$$
\mathbf{X}_{1}^{n+1}=\mathbf{L}_{1}^{-1} \mathbf{R}_{m} \cdot \mathbf{L}_{m}{ }^{-1} \mathbf{R}_{m-1} \mathbf{L}_{m-1}{ }^{-1} \mathbf{R}_{m-2} \cdots \mathbf{L}_{2}{ }^{-1} \mathbf{R}_{1} \mathbf{X}_{1}^{n} \triangleq \mathbf{T}(\omega) \mathbf{X}_{1}^{n}
$$

where $\mathbf{T}(\omega)=\mathbf{L}_{1}{ }^{-1} \mathbf{R}_{m} \cdot \mathbf{L}_{m}{ }^{-1} \mathbf{R}_{m-1} \mathbf{L}_{m-1}{ }^{-1} \mathbf{R}_{m-2}{ }^{\cdots} \mathbf{L}_{2}{ }^{-1} \mathbf{R}_{1}$ is the transfer matrix.

In this case, the Bloch theorem[14] can be used on the boundaries between the neighborhood microstructures, as follows 


$$
\mathbf{X}_{1}^{n+1}=e^{\mathrm{j} q a} \mathbf{X}_{1}^{n}
$$

where $q$ is the longitudinal wave vector, valuing in the first Brillouin district, $a$ the lattice constant of one microstructure, and $a=\sum_{i=1}^{m} l_{i}$.

Simultaneity of Eq. 11 and Eq. 12 leads to

$$
\left(\mathbf{T}(\omega)-e^{\mathrm{j} q a} \mathbf{I}\right) \mathbf{X}_{1}^{n}=\mathbf{0}
$$

where $\mathbf{I}$ is identity matrix of size $2 \times 2$.

For a given vibration angular frequency $\omega$, to make $\mathbf{X}_{1}^{n}$ a non-zero solution, the characteristic equation must be

$$
\left|\mathbf{T}(\omega)-e^{\mathrm{j} q a} \mathbf{I}\right|=0
$$

By solving Eq. 14, the corresponding relationship between the wave vector and the vibration angular frequency can be drawn as the band structure diagram of the longitudinal vibration of the 1D PC bar.

Characteristics of bipropellant rod-shaped structures of 1D PCs. For the perfect bipropellant rod-shaped structures of 1D PCs as shown in Fig.2(b), the lattice constant of its microstructure is assumed as $a=0.2 \mathrm{~m}$. The cross section is round with the radius $r=20 \mathrm{~mm}$. Each microstructure consists of two materials, aluminum and epoxy (which are common in engineering and tractable). And the material parameters are shown in Table 1. Base on TMM, the longitudinal vibration band gaps of the rod-shaped structures of 1D PCs is simulated. And the diagram of energy band structure is shown in Fig. 4.

Table 1 Material parameters

\begin{tabular}{ccc}
\hline Material & $\begin{array}{c}\text { Density } \\
\rho\end{array}$ & $\begin{array}{c}\text { Elasticity } \\
\text { modulus } E\end{array}$ \\
& {$\left[\mathrm{~kg} / \mathrm{m}^{3}\right]$} & {$\left[10^{10} \mathrm{~Pa}\right]$} \\
\hline Aluminum & 2730 & 7.76 \\
Epoxy & 1180 & 0.435 \\
\hline
\end{tabular}

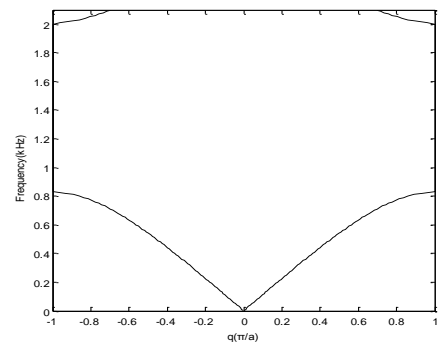

(a) real wave vector

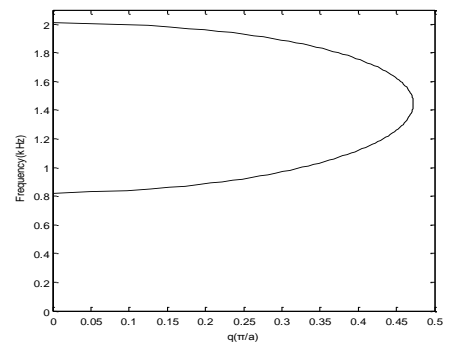

(b) virtual wave vector Figure 4. Longitudinal vibration energy band structure

From Fig.4, there are none curves of real wave vector during some frequency range in the band structure diagrams, which indicates that vibration waves of these frequency cannot propagate on the rod-shaped structure of 1D PCs. The range of these frequency is called band gap, validating the effectiveness on suppression of vibration propagation on the designed rod-shaped structures. Furthermore, the corresponding amplitude of imaginary wave vector during band gaps reflect the capability on vibration suppression.

The simulation results of energy band structure show that the starting and terminating frequencies(SF and TF) of the first gap of longitudinal vibration are $840 \mathrm{~Hz}$ and $2000 \mathrm{~Hz}$ respectively. Moreover, the first and second natural frequencies of longitudinal vibration of one single microstructure are respectively $962 \mathrm{~Hz}$ and $2628 \mathrm{~Hz}$. It can be suggested that gaps of local resonant PCs are closely related to its microstructure configuration. Thus with the design of microstructure configuration, the natural frequencies of microstructure are changed, adjusting gaps covering the frequency range of interest.

The larger the lattice constant is, the lower the natural frequencies of microstructure are, thus the low-frequency band gap appearing. Fig. 5 displays the variation of the first band gap and the second band gap of the 1D PCs as a function of the factor $a$. During the calculations, the radius $r$ is fixed to be $0.02 \mathrm{~m}$ and the length ratio is $a_{A l} / a_{E p o x y}=1$. As shown in Fig. 5, with the increasing of the lattice 
constant, both the first and the second band gaps move to low-frequency observably, but the width of both gaps decrease at the same time. Meanwhile, SF of the first gap and TF of the second gap move closer, which means there might be a new blended gap equivalently.

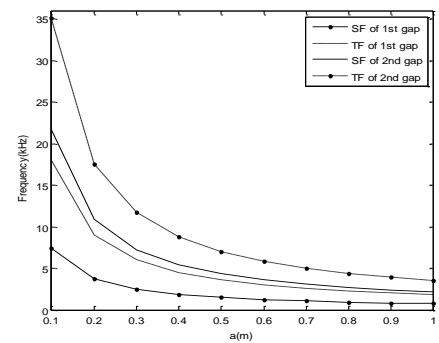

Figure 5. The first and the second band gaps with the change of the lattice constant

The length ratio also has effect on the natural frequencies of the microstructure, which can adjust the range and width of band gaps. The variation of the first band gap and the second band gap as a function of the factor $a_{A l} / a_{E p o x y}$ is shown as Fig. 6(a) and Fig. 6(b) illustrates the width variety of the two gaps. In the Fig. 6, the lattice constant $a$ is fixed to be $0.9 m$ with the radius fixes to $r=0.02 m$. From Fig. 6(a), it can be found that with the increase of the ratio of Aluminum, both gaps move to high-frequencies. That is by reason that the natural frequencies of an Aluminum bar are correspondingly higher than those of an Epoxy bar of the same geometry and boundary conditions. As is shown in Fig. 6(b), the width of the first band gap increases to maximum which is around $2 \mathrm{kHz}$ and then drops again. Unlike the trend of the first gap, the second gap fluctuates twice, and it reaches vale where the first gap achieves peak, which might due to the differences on the leading vibrate models. Therefore, it is illuminated that the width of band gaps can be adjusted by proper design of the ratio. It also seems that the band gaps close where the ratio is ultimate at 0 and $\infty$, as shown in Fig. 7(a) and (b), validating the fact that a rod made of unitary material does not have band gaps. That is, the material properties can affect the characters of gaps, but cannot determine the existence of gaps. Besides, the sequence of the two materials in one microstructure are irrelevant with gaps, as shown in Fig. 8.

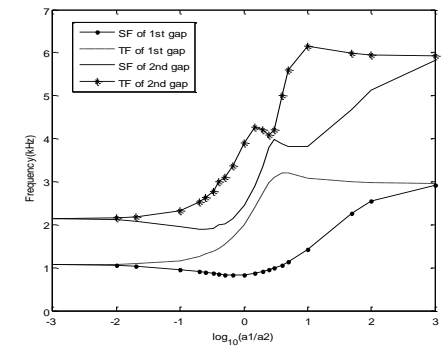

(a) gap range

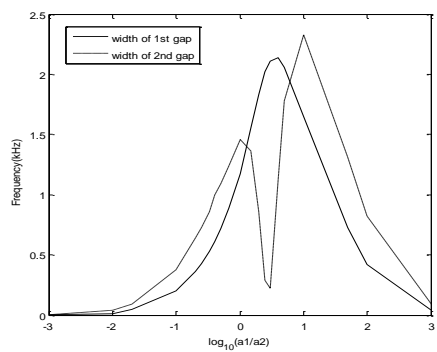

(b) gap width

Figure 6. Characteristics of gaps as a function of ratio

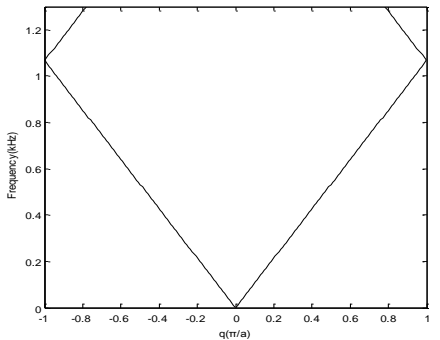

(a) $a_{A l} / a_{E p x y y}=0$ (pure Epoxy bar)

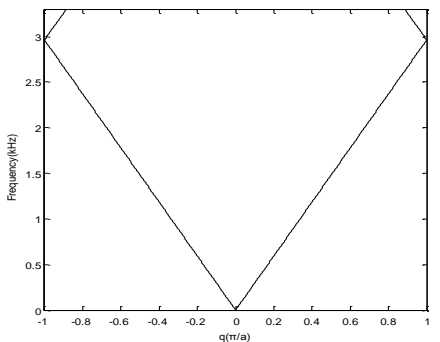

(b) $a_{A l} / a_{E p o x y}=\infty$ (pure

Aluminum bar)

Figure 7. Band structure diagram for a bar made of unitary material

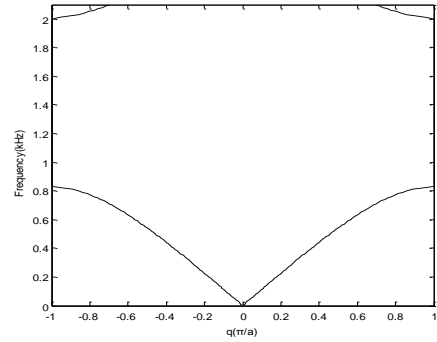

(a) Al-Epoxy

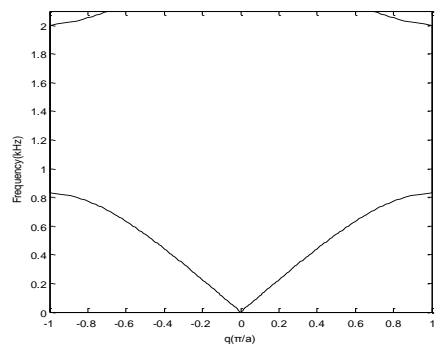

(b) Epoxy-Al

Figure 8. Effect of sequence of the two materials in one microstructure $\left(a_{N} / a_{\text {Eman }}=1\right)$ 
Characteristics of finite 1D PC rods. For the infinite bipropellant rods of 1D PCs, the sequence of different materials in one microstructure has no impact on gaps due to the periodic boundary conditions. However, in practical applications, the dimension of a component is finite. The number of microstructures and the sequence of materials will ineluctably infect the characteristics of gaps.

Fig. 10 is the sketch of finite bipropellant rods of 1D PCs. The number of microstructures is assumed as 3. The configuration parameters of the microstructure and the material parameters are the same with those of infinite rod-structuers. The sine excitation of acceleration is imposed at one end of the rod, with the input amplitude of 1 and the frequency range for $10-2000 \mathrm{~Hz}$. Then output amplitude of acceleration at the other end is observed, as shown in Fig. 10.

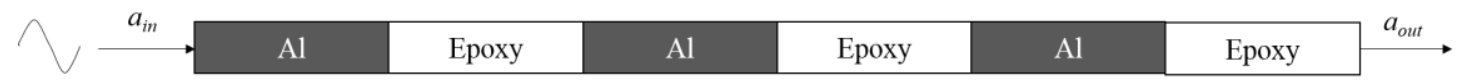

Figure. 10 Sketch of finite 1D bipropellant PC rod

With the other conditions fixed, the frequency response curves of the finite 1D PC rod with different microstructure numbers and sequence of materials in one microstructure is simulated respectively, as shown in Fig.11 and 12. As is shown in Fig. 11, the sequence of materials in on microstructure do has effect on gaps of the finite 1D PC rods. But with the amount of microstructures increasing, the finite 1D PC rod verges on the infinite one, resulting that gaps are less sensitive to the sequence. Theoretically, finite PC rod with larger number of microstructures has stronger capacity of vibration suppression. Moreover, it is less sensitive to the sequence of materials in one microstructure, which means the capacities of propagation reduction for both positive and negative direction vibration wave are almost equal. Thus the direction of the installation has no impact in application.

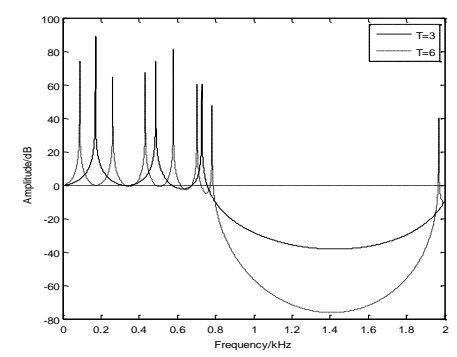

Figure 11. Frequency response of the

finite 1D PC rods with different microstructure numbers

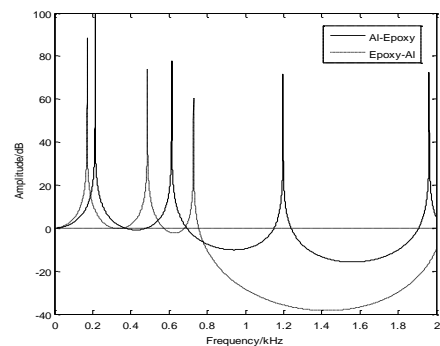

(a) 3 microstructures

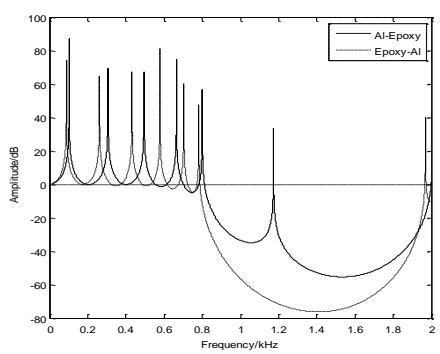

(b) 6 microstructures

Figure 12. Frequency response of the finite 1D PC rods with different sequences

\section{Research on high-frequency vibration isolation for rod-shaped structures}

As shown in Fig.1(a), taking the truss rod of support structures of space camera as an example, the method of high-frequency vibration isolation is studied.

Parametric design of 1D PCs for high-frequency vibration isolation. According to the research above and the requirements of high-frequency vibration isolation, the microstructure is designed as the $1 \mathrm{D}$ bipropellant straight rod with circular cross-section. And the lattice constant is $a=0.9 \mathrm{~m}$, with the radius $r=20 \mathrm{~mm}$ and the ratio $a_{A l} / a_{E p o x y}=1$. Each microstructure consists of two materials, Aluminum and Epoxy are shown in Table 1 . Then the SF and TF are respectively $830 \mathrm{~Hz}$ and $2010 \mathrm{~Hz}$, and the frequency band range of vibration isolation is wide.

Theoretically, the larger the lattice constant is, the stronger the capacity of vibration reduction is, but due to the limitation of dimension in realistic application, the unit number is 3 , with the overall length of $2.7 \mathrm{~m}$, which is exactly the length of the original truss rods, as shown in Fig. 13.

\begin{tabular}{|l|llll}
\hline Epoxy & $\mathrm{Al}$ & $\mathrm{Al}$ & $\mathrm{Epoxy}$ & $\mathrm{Al}$ \\
\hline
\end{tabular}

Figure. 13 Sketch of the vibration isolation rod base on 1D PCs 
Simulation verification and analysis. Inspiriting respectively at the Epoxy end and the Aluminum end of the designed rod as well as one end of the rod made of Aluminum only from $10 \mathrm{~Hz}$ to $2000 \mathrm{~Hz}$, the acceleration frequency response of the other end is simulated, as shown in Fig.14 and 15. Compared with Fig.14, it can be indicated that the vibration wave propagation cannot be suppressed when the pure Aluminum rod is in the state of non-control. While under the same conditions, the high-frequency vibration isolation appears on the designed rod. This shows that the design of the PC rod is effective in high-frequency vibration, but sensitive to the sequence of materials in one microstructure, which means the capacities of propagation reduction for vibration wave of both positive and negative direction are quite different. Thus the Epoxy end must be installed close to disturbing sources while the Aluminum end towards the target equipments of vibration suppression.

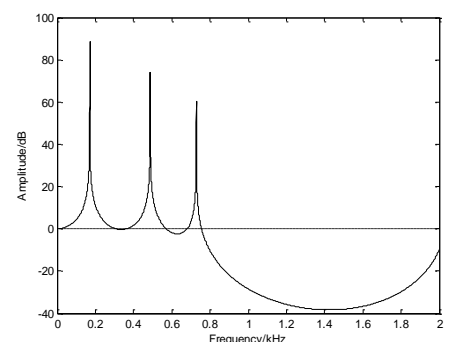

(a) Inspiriting at the Epoxy end

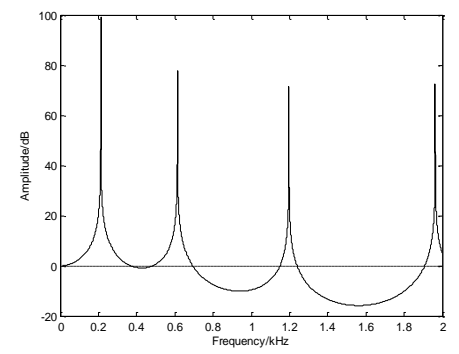

(b) Inspiriting at the Aluminum end

Figure.14 Frequency response curves of the designed rods

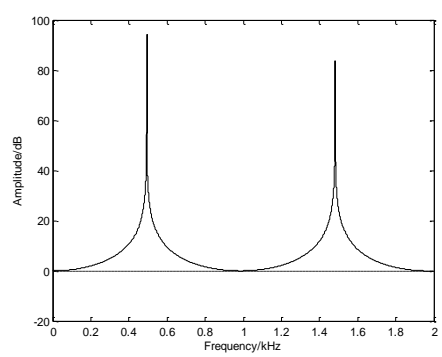

Figure.15 Frequency response curve of rod made of Aluminum only

\section{Conclusions}

This paper conducts the research on rod-shaped structures of 1D PCs by TMM. The characteristics of gaps are studied, and the capacity of vibration isolation of finite rod of 1D PCs is analyzed combined with the frequency response curves. Taking the truss rod of support structure of space camera as an example, this paper presents a design of bipropellant rods based on 1D PCs and simulation is carried out. Results show that the proposed rod can suppress the propagation of vibration of $750-2000 \mathrm{~Hz}$, validating the method of high-frequency vibration isolation for rod-shaped structures base on 1D PCs, which provides a new approach to high-frequency vibration isolation.

\section{References}

[1] L. Zhang, X.Zh. Jia. Design and optimization of trussed supporting structure for off-axis three-mirror reflective space camera. Optics and Precision Engineering,2009,3(17) (in Chinese)

[2] J.P. Jiang, D.X. Li. Research on finite element modeling and vibration control for smart solar array. Journal of Dynamics and Control,2009,7(2) (in Chinese)

[3] Y. Tian. Study on vibration characteristic for space truss structure of spacecraft. Harbin Institute of Technology,2012,12(in Chinese)

[4] M. S. Kushwaha et al. Acoustic band structure of periodic elastic composites. Phys. Rev. Lett., 1993, 71: 2022-2025

[5] S. Benchabane, A. Khelif, J-Y. Rauch, L. Robert, V. Laude, Phys. Rev. E 73 (2006)065601.

[6] A. Khelif, B. Aoubiza, S. Mohammadi, A. Adibi, V. Laude, Complete band gaps in two-dimensional phononic crystal slabs, Physical Review E74 (2006) 5.

[7] Z.Y. Liu, X.X. Zhang, Y.W. Mao, Y.Y. Zhu, Z.Y. Yang, C.T. Chan, P. Sheng, Locally resonant sonic materials,Science289 (2000) 1734-1736. 
[8] Cervera F., Sanchis L., Sanchez-Perez J. V., et al. Refractive acoustic devices for airborne sound[J]. Phys. Rev. Lett., 2002,(2) :023902-023904.

[9] Mei J, Ma G, Yang M, et al. Dark acoustic metamaterials as super absorbers for low-frequency sound[J]. Nature Communications, 2012, 3: 756.

[10] J. H. Wen, D. L. Yu, Y. Xiao, etc.. Propagation of elastic waves in artificial periodic structures. Science Press, Beijing, 2015. (in Chinese)

[11]D.L. Yu, Y.Z. Liu, J Reng, etc..Vibration chatacterisctiscs and simulation of one-demensional phononic crystals.Journal of Vibration and Shock,2005,24(2)

[12] Przemysław Nowak, Maciej Krawczyk. Phononic Band Gaps in One-dimensional Phononic Crystals with Nanoscale Periodic Corrugations at Interfaces. Computational Methods in Science and Technology 16(1), 85-95 (2010)

[13] D. X. Li. Advanced structural dynamics. Science Press, Beijing, 2013

[14]L. Brillouin, Wave propagation in periodic structures, McGraw-Hill Book Company, New York, 1946 